\title{
Logical recoding of $S-R$ rules can reverse the effects of spatial $S-R$ correspondence
}

\author{
Peter WÜhr ANd RUPert Biebl \\ Friedrich-Alexander-Universität Erlangen-Nürnberg, Erlangen, Germany
}

\begin{abstract}
Two experiments investigated competing explanations for the reversal of spatial stimulus-response (S-R) correspondence effects (i.e., Simon effects) with an incompatible S-R mapping on the relevant, nonspatial dimension. Competing explanations were based on generalized S-R rules (logical-recoding account) or referred to display-control arrangement correspondence or to S-S congruity. In Experiment 1, compatible responses to finger-name stimuli presented at left/right locations produced normal Simon effects, whereas incompatible responses to finger-name stimuli produced an inverted Simon effect. This finding supports the logical-recoding account. In Experiment 2, spatial S-R correspondence and color S-R correspondence were varied independently, and main effects of these variables were observed. The lack of an interaction between these variables, however, disconfirms a prediction of the display-control arrangement correspondence account. Together, the results provide converging evidence for the logical-recoding account. This account claims that participants derive generalized response selection rules (e.g., the identity or reversal rule) from specific S-R rules and inadvertently apply the generalized rules to the irrelevant (spatial) S-R dimension when selecting their response.
\end{abstract}

The mapping of stimuli onto responses is a major determinant of behavior, a phenomenon called stimulusresponse ( $\mathrm{S}-\mathrm{R})$ compatibility. Research on $\mathrm{S}-\mathrm{R}$ compatibility has gained important insights into the mechanisms of human response selection (see Proctor \& Vu, 2006, for an overview). Much research has focused on the effects of spatial S-R compatibility, investigating how the spatial arrangement of stimuli and responses affects the speed and quality of behavior (e.g., Hommel \& Prinz, 1997). The general result of these investigations has been that spatial correspondence of stimulus and response locations produces better performance in terms of speed and accuracy than does spatial noncorrespondence. This general rule holds when stimulus location is relevant for selecting the correct response, a finding called spatial $S-R$ compatibility proper. The rule also holds when stimulus location is irrelevant for selecting the correct response, a finding denoted as the Simon effect. The present study is concerned with a striking exception to this general rule: a reversal of the Simon effect that appears with an incompatible mapping on the relevant S-R dimension.

The Simon effect is particularly interesting because it suggests that stimuli activate spatially corresponding responses in an automatic fashion (e.g., Zorzi \& Umiltà, 1995). In a typical Simon task, participants press a leftside key to a green stimulus and a right-side key to a red stimulus, and stimuli appear unpredictably at a left or at a right location. The finding of faster and more accurate responses to spatially corresponding conditions (e.g., green $\mathrm{S}$ at left location) than to spatially noncorresponding con- ditions (e.g., green $\mathrm{S}$ at right location) has been called the Simon effect, because J. R. Simon was the first to describe the phenomenon (e.g., Simon \& Rudell, 1967; see Proctor $\& \mathrm{Vu}, 2006$, chap. 4, for a review).

The most prominent accounts of the Simon effect distinguish between two routes of response activation (e.g., Kornblum, Hasbroucq, \& Osman, 1990; Zhang, Zhang, \& Kornblum, 1999). A controlled route of processing is assumed to determine the correct response on the basis of the relevant stimulus attribute (e.g., color, in the example above) and the S-R mapping rule. An automatic route of processing is assumed to simultaneously activate the spatially corresponding response in the response set on the basis of stimulus location. Both routes converge on the correct response in spatially corresponding conditions, and this response is quickly selected and executed. By contrast, the routes activate different responses in spatially noncorresponding conditions, and the resulting response conflict produces a cost in time and/or accuracy.

Hedge and Marsh (1975) reported an important exception to the general rule that performance is better with spatially corresponding than with spatially noncorresponding conditions. These authors investigated how $\mathrm{S}-\mathrm{R}$ compatibility on the relevant, nonspatial S-R dimension affects location processing in the Simon task. Participants responded to the color of a stimulus by moving their finger from a centrally located home key to a response key on the left or right side of the home key. The left and right response keys were also colored, allowing Hedge and Marsh to manipulate the mapping of stimulus to response key

P.Wühr,wuehr@fk14.tu-dortmund.de 
colors. In the compatible condition, participants had to press the green key to the green stimulus and the red key to the red stimulus. By contrast, in the incompatible condition, participants had to press the red key to the green stimulus and the green key to the red stimulus. With the compatible mapping, Hedge and Marsh observed the typical Simon effect; that is, reaction times (RTs) were shorter by $23 \mathrm{msec}$ in corresponding than in noncorresponding conditions. In contrast, with the incompatible mapping, a reversed Simon effect occurred, in that RTs were shorter by $55 \mathrm{msec}$ in noncorresponding than in corresponding conditions. This so-called Hedge and Marsh reversal of the Simon effect has been replicated several times since then (e.g., Arend \& Wandmacher, 1987; Lu \& Proctor, 1994; Wühr, 2004).

Hedge and Marsh (1975) offered an explanation for their findings in terms of logical-recoding operations in S-R translation. The authors assumed that with the compatible mapping of S-R colors, participants determined the correct response on the basis of an identity rule, which says something like "respond with the key that matches the stimulus." By contrast, with the incompatible color mapping, participants were assumed to determine the correct response on the basis of a reversal rule, which says something like "respond with the key that does not match the stimulus." In order to explain the pattern of Simon effects, the authors suggested that the logical-recoding rule used for the relevant $\mathrm{S}-\mathrm{R}$ dimension (i.e., color) is inadvertently applied to the irrelevant $\mathrm{S}-\mathrm{R}$ dimension (i.e., location) as well. Thus, with the compatible mapping, the identity rule would activate a response with the same color at the corresponding location, producing a normal Simon effect. With the incompatible mapping, the reversal rule would activate a response with a different color at the noncorresponding location, producing an inverted Simon effect (see De Jong, Liang, \& Lauber, 1994, for an extension of this account).

Simon, Sly, and Vilapakkam (1981) proposed an alternative explanation for the Hedge and Marsh reversal of the Simon effect in terms of the visual correspondence between display and control features. These authors noted that in the Hedge and Marsh task, performance is better when the stimulus appears on the side of the congruently colored key than when the stimulus appears on the side of the incongruently colored key. The former situation appears in spatially corresponding conditions with a compatible S-R mapping (Figures 1A and 1B) and in spatially noncorresponding conditions with an incompatible S-R mapping (Figures $1 \mathrm{G}$ and $1 \mathrm{H}$ ). The latter situation appears in spatially noncorresponding conditions with a compatible S-R mapping (Figures $1 \mathrm{E}$ and $1 \mathrm{~F}$ ) and in spatially corresponding conditions with an incompatible S-R mapping (Figures 1C and 1D). Simon et al. (1981) denoted this relationship as the display-control arrangement correspondence.

In the experimental design used by Hedge and Marsh (1975), logical-recoding operations and display-control arrangement correspondence are confounded. Simon et al. (1981) conducted an experiment in which they attempted to isolate the effects of logical recoding. In their Experiment 3, participants responded to the color of a stimulus presented at a central location by pressing a green key at a left location or a red key at a right location. Simultaneously with the visual stimulus, an irrelevant tone occurred at either the left or the right ear. The compatible S-R mapping condition showed a regular Simon effect of
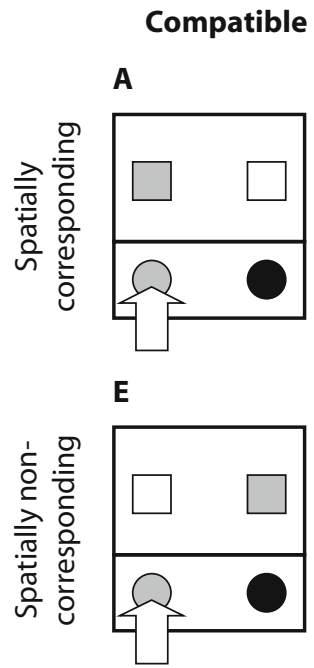

$\mathbf{F}$

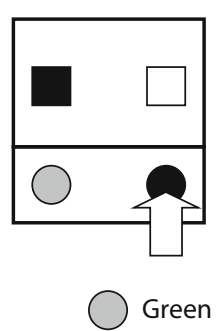

B
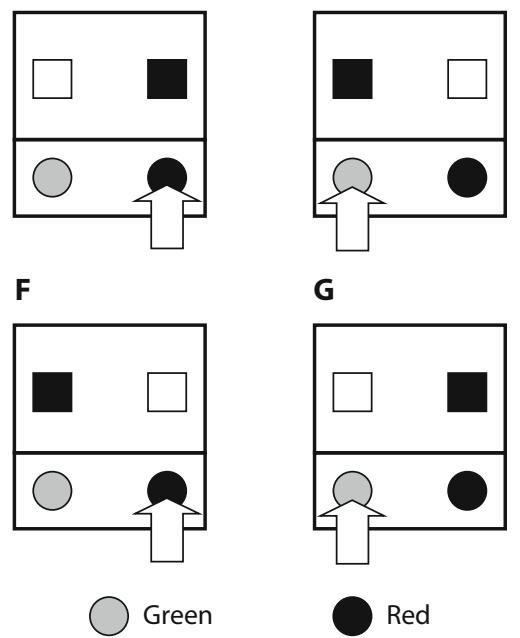

G

Incompatible S-R Mapping

C
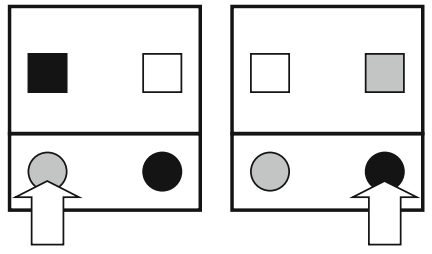

H

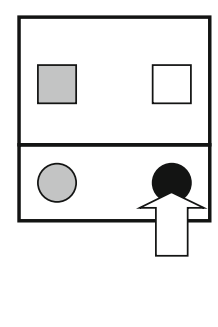

Figure 1. Different experimental conditions in the task used by Hedge and Marsh (1975, Experiment 1). Participants responded to the color (green vs. red) of a stimulus (depicted by squares) by pressing a key of the same color (compatible stimulusresponse [S-R] mapping) or by pressing a key of the opposite color (incompatible $S-R$ mapping). An arrow denotes the correct key in each condition. 
more than $50 \mathrm{msec}$, but there was no Simon effect with the incompatible S-R mapping. The authors interpreted their failure to obtain the reversed Simon effect with the incompatible $\mathrm{S}-\mathrm{R}$ mapping as evidence against the logical-recoding account. Simultaneously, the absence of a correspondence effect with the incompatible mapping is consistent with their account because there was no display-control arrangement correspondence between the central visual stimulus and the lateral response keys in their experiment.

In a more recent study, Proctor and Pick (2003) investigated the conditions under which the Hedge and Marsh reversal of the Simon effect can occur in order to decide between competing explanations. They reported three main findings and interpreted these findings as supporting the display-control arrangement correspondence hypothesis. First, when the relevant S-R dimension was color and the location of a tone provided the irrelevant location stimulus, as in Experiment 3 in Simon et al. (1981), Proctor and Pick observed positive Simon effects with the compatible S-R mapping but no effects with the incompatible S-R mapping, replicating the results of Simon and colleagues. Second, when the relevant S-R dimension was color and the location of the visual stimulus provided the irrelevant location information, as in the original Hedge and Marsh task, the reversal of the Simon effect with an incompatible $\mathrm{S}-\mathrm{R}$ mapping depended on a clearly visible color labeling of the response keys. Third, when the relevant S-R dimension was visual location and the location of a tone provided the irrelevant location stimulus, Proctor and Pick observed a small but significant reversal of the Simon effect with the incompatible S-R mapping. In their recent book, Proctor and $\mathrm{Vu}$ (2006) summarized the empirical situation as follows: "The Hedge and Marsh reversal with colored stimuli apparently depends on the response keys being visibly labeled, and the reversal does not occur when the irrelevant location information is conveyed by tones; this points toward display-control arrangement correspondence as a critical factor in the Hedge and Marsh reversal" (p. 127).

The purpose of the present study is to provide both theoretical arguments and new empirical data for the notion that logical-recoding operations, and not display-control arrangement correspondence, play the critical role in the Hedge and Marsh reversal of the Simon effect. We first will discuss the two empirical findings that Proctor and Vu (2006) regarded as the major facts against logical recoding, and then we will address a yet neglected empirical finding against display-control arrangement correspondence. According to Proctor and $\mathrm{Vu}$, dependence of the Hedge and Marsh reversal on a visible labeling of the response keys in the original task supports display-control arrangement correspondence because "there is no reason why logical recoding should depend on visible color labels, whereas display-control arrangement correspondence would seem to require visible response labels that can be matched to the colored stimuli" (p. 127). In contrast to this view, we see a clear reason why logical recoding should depend on visible color labels, and the reason is that visibility of color labels should affect the features used for representing the responses. In particular, when the color labels are not visible, it is very likely that participants code the responses in terms of locations, and not in terms of "colors," because location is visible and color is not. As a result, most participants will recode the $\mathrm{S}-\mathrm{R}$ mapping from a color-to-color to a color-to-location mapping. This interpretation is supported by the fact that Proctor and Pick (2003) observed much smaller effects of S-R mapping with invisible response labels (Experiment 1, $6 \mathrm{msec}$; Experiment 2, approximately $40 \mathrm{msec}$ ) than with visible labels (Experiment 3, approximately $90 \mathrm{msec}$ ).

The second argument put forward in favor of displaycontrol arrangement correspondence is the failure to observe a reversal of the Simon effect when the color of a centrally presented stimulus is mapped incompatibly onto response color and a tone delivers the irrelevant location stimulus (Proctor \& Pick, 2003, Experiment 3; Simon et al., 1981, Experiment 3). It should first be noted that in this task, the typical finding is a significantly larger Simon effect with the compatible than with the incompatible S-R mapping, although the effect does not reverse with the incompatible mapping. A possible explanation for this finding in terms of logical-recoding operations is that the inadvertent application of logical-recoding operations to the irrelevant $\mathrm{S}-\mathrm{R}$ dimension is more likely when the same object (or event) delivers the relevant color and the irrelevant location information than when different objects (or groups) deliver the information. This interpretation is consistent with our recent finding of much smaller Simon effects when different objects provide relevant and irrelevant information, respectively, as compared with the integrated version of the Simon task (Wühr, Biebl, Umiltà, \& Müsseler, in press). Second, this interpretation is also consistent with numerous findings demonstrating that congruency effects are significantly reduced when relevant and irrelevant information are part of different perceptual objects or groups than when they are part of the same object or group (e.g., Kramer \& Jacobsen, 1991; Wühr \& Waszak, 2003). Third, this interpretation is also consistent with the observation of a small, but significant, reversal of the Simon effect when the variable (left/right) location of a visual stimulus is incompatibly mapped onto response location and a tone delivers an irrelevant location stimulus (Proctor \& Pick, 2003, Experiments 1 and 4). For this case, one might argue that the logical-recoding rule used for response selection is applied to the irrelevant stimulus location when the tone and the visual stimulus appear on the same side and are, therefore, perceptually integrated.

Several studies have demonstrated the Hedge and Marsh reversal of the Simon effect with displays for which display-control arrangement correspondence cannot play a role. Arend and Wandmacher (1987, Experiment 1), for example, found a positive Simon effect when left- and right-pointing arrows, presented to the left or right of fixation, were compatibly mapped to left/right responses and an inverted Simon effect when the arrows were incompatibly mapped to left/right responses (see also Lu \& Proctor, 1994, Experiment 3). In their Experiment 2, Arend and Wandmacher observed a similar pattern of results when the words left or right were used instead of arrows (see also Lu \& Proctor, 1994, Experiment 1). The Hedge 


\section{Compatible S-R Mapping}

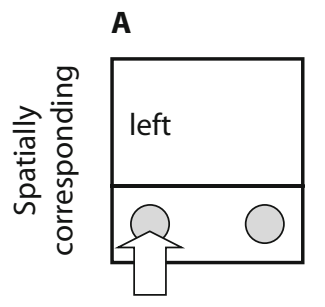

E

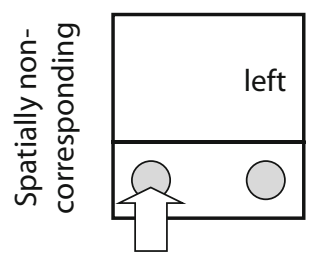

B

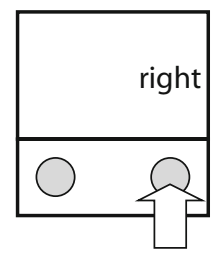

$\mathbf{F}$

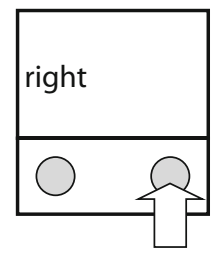

Incompatible S-R Mapping

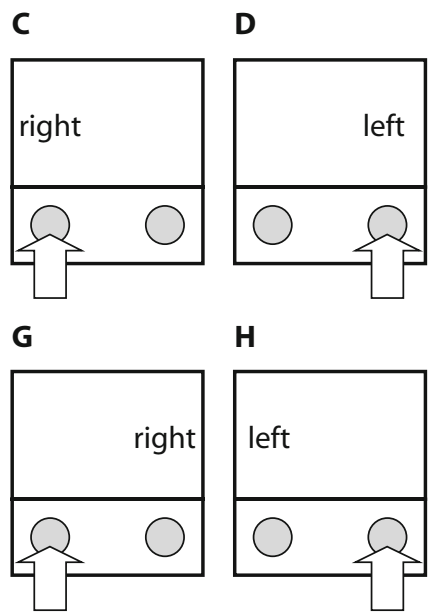

Figure 2. Different experimental conditions in the task used by Arend and Wandmacher (1987, Experiment 2). Participants responded to the meaning of location words ("left" vs. "right") by pressing a key at the corresponding location (compatible stimulus-response [S-R] mapping) or by pressing a key at the opposite location (incompatible $S-R$ mapping). An arrow denotes the correct key in each condition.

and Marsh reversal obtained with arrow and word stimuli cannot be explained on the basis of display-control arrangement correspondence, because the visual stimuli do not share perceptual features with the responses. Unfortunately, the results cannot unequivocally be attributed to logical recoding, because the findings might also be explained on the basis of S-S congruity effects (e.g., Hasbroucq \& Guiard, 1991; Simon \& Sudalaimuthu, 1979). Performance is better when the two aspects of the visual stimulus are congruent (Figure 2, panels A/B and G/H) than when the two aspects of the visual stimulus are incongruent (Figure 2, panels C/D and E/F). To conclude, at present, unequivocal empirical evidence exists neither for display-control arrangement correspondence nor for logical-recoding operations as a factor in the Hedge and Marsh reversal of the Simon effect.

The empirical goal of the present study was to investigate both logical-recoding operations and display-control arrangement correspondence in isolation. Experiment 1 was designed to study the possible effects of logicalrecoding operations on the Simon effect without being confounded with possible effects of display-control arrangement correspondence or S-S congruity. In Experiment 1, participants responded to the German words for "index finger" or "middle finger" on the basis of a compatible or an incompatible S-R mapping. The words randomly appeared to the left or right of fixation, and therefore, irrelevant stimulus location and response location were corresponding or noncorresponding. The observation of a normal Simon effect with the compatible S-R mapping and of an inverted Simon effect with the incompatible S-R mapping would provide strong evidence for the application of logical-recoding operations in this task.

Experiment 2 was designed to study the possible effects of display-control arrangement correspondence in isolation. In this experiment, participants responded to geometrical shapes (i.e., diamond vs. square) by pressing a key with the left or the right hand. Because stimuli randomly appeared to the left or right of fixation, a Simon effect of spatial S-R correspondence was to be expected. In addition, we manipulated display-control arrangement correspondence with regard to color. The stimulus randomly appeared in green or red color, and the participants wore a green glove on one hand and a red glove on the other hand. The display-control arrangement correspondence hypothesis predicts an interaction between the effects of spatial S-R correspondence and color S-R correspondence in this task.

\section{EXPERIMENT 1}

The purpose of Experiment 1 was to isolate the effects of logical-recoding operations in the Simon task. Therefore, we devised a task in which effects of display-controlarrangement correspondence and S-S congruity were unlikely to appear. In our task, participants responded to the German words for "index finger" or "middle finger" on the basis of a compatible or an incompatible S-R mapping. In particular, with the compatible mapping, the participants performed a keypress with the finger denoted by the word; with the incompatible mapping, the participants performed a keypress with the finger not denoted by the word. In addition to mapping, we also varied spatial $\mathrm{S}-\mathrm{R}$ correspondence between horizontal stimulus location on the computer screen and the relative location of the to-be-pressed key (i.e., index finger $\rightarrow$ left key, middle finger $\rightarrow$ right key). Because the participants could represent both the compatible and the incompatible S-R mapping in terms of logical-recoding operations (i.e., the identity vs. reversal rule), we expected a positive Simon effect 
with the compatible mapping and an inverted Simon effect with the incompatible S-R mapping.

The present task should be immune against effects of display-control arrangement correspondence because the visual stimuli (i.e., the words "index finger" and "middle finger") do not share visible features with the responses. Moreover, the present task should be less sensitive to the effects of S-S congruity than tasks in which location words or arrowheads are presented at congruent or incongruent locations. Finger names should not have strong associations with left or right locations, because they have opposite relative locations on the left and the right hand, respectively.

\section{Method}

Participants. Sixteen volunteers ( 9 of them female, 7 male), mostly students of the Friedrich Alexander University, with a mean age of 24.4 years (range, 20-31 years), participated for payment (€3) or course credit. In this and the following experiments, the participants were naive with respect to the purpose of the study and classified themselves as having normal (or corrected-to-normal) visual acuity.

Apparatus and Stimuli. The participants sat in a dimly lit room in front of a 17-in. color monitor, with an unconstrained viewing distance of approximately $60 \mathrm{~cm}$. A computer program written in ERTS language (BeriSoft, Frankfurt am Main, Germany) controlled stimulus presentation and response registration. All responses were keypresses on the "open menu" key and the right "control" key, which are in adjacent locations on a standard computer keyboard. The participants operated the "open menu" key with the index finger and the "control" key with the middle finger of the right hand.

A plus sign served as a fixation point at screen center. Visual stimuli were the uppercase words Mittelfinger (German for "middle finger") and Zeigefinger (German for "index finger"), which were presented in two parts (one above the other). Each word subtended about $30 \times 12 \mathrm{~mm}$ (about $2.9^{\circ} \times 1.2^{\circ}$ ). The words were presented $60 \mathrm{~mm}$ (about $5.7^{\circ}$ ) to either the left or the right of fixation. All the visual stimuli were presented in white on a black background.

Procedure. The participants performed with a compatible S-R mapping in the first part of the experiment and with an incompatible S-R mapping in the second part of the experiment, or vice versa. Each part of the experiment had the following structure. First, the instructions appeared on the screen, and the participants could read them at leisure. With the compatible mapping, the participants were instructed to respond with the index finger to the word "index finger" and with the middle finger to the word "middle finger." With the incompatible mapping, the participants were instructed to respond with the index finger to the word "middle finger" and with the middle finger to the word "index finger." In both cases, the participants were also informed that the stimuli would randomly appear at different locations but that location was irrelevant for the task. Next, the participants could practice for a block of 22 trials. Then the participants worked through eight blocks of 22 experimental trials each. The first 2 trials in each block were warm-up trials and were not recorded. The participants could take short rests between blocks. The whole experiment took about $20 \mathrm{~min}$.

The sequence of events in an individual trial was as follows. First, there was a blank (i.e., empty) screen for $500 \mathrm{msec}$. Then the fixation point was shown for $500 \mathrm{msec}$. Next, the imperative stimulus was added to the display and remained there for $500 \mathrm{msec}$. Stimulus offset was followed by another blank period of $500 \mathrm{msec}$. Each stimulus appeared randomly to the left or right of fixation. With the onset of the imperative stimulus, the computer started to record keypresses and RTs for a period of $1,000 \mathrm{msec}$. There were five repetitions for each combination of stimulus word (Zeigefinger, Mittelfinger) and stimulus location (left, right) in each block of 20 trials.

In each task, two types of errors produced an error message that was presented for $2,000 \mathrm{msec}$ at screen center. First, the participants received an error message when they had pressed the wrong key within the registration period of $1,000 \mathrm{msec}$. Second, the participants were told to respond more quickly when no response had appeared within the registration period.

Design. Experiment 1 rested on a $2 \times 2$ within-subjects design. The first factor concerned the mapping between the finger name stimuli and the response finger, which was either compatible or incompatible (see the Procedure section). The second factor concerned spatial $S-R$ correspondence between the location of the finger name stimulus on the screen and the relative location of the response finger. This relationship was either spatially corresponding (i.e., "index finger" appeared at the left location, "middle finger" appeared at the right location) or noncorresponding (i.e., "index finger" appeared at the right location, "middle finger" appeared at the left location). Dependent variables were RTs of correct responses and the percentages of errors (PEs).

\section{Results}

To eliminate outliers, we removed all trials on which RTs differed from the individual mean by more than three standard deviations. This procedure eliminated $0.90 \%$ of the trials with the compatible mapping and $0.50 \%$ of the trials with the incompatible mapping. Initial analyses showed that the order of S-R mappings did not affect the results in RTs or in error rates (all interactions had $F$ values $<1.6$ and $p$ values $>.20$ ). RTs and PEs observed in Experiment 1 are listed in Table 1.

Reaction times. RTs from error-free trials were subjected to an ANOVA for repeated measurements designs, with mapping and S-R correspondence as within-subjects variables. The main effect of mapping was significant $\left[F(1,15)=33.80, M S_{\mathrm{e}}=2,125.65, p<.001\right]$. The participants responded more quickly with the compatible than with the incompatible mapping of finger name stimuli to response fingers (550 vs. $617 \mathrm{msec})$. The main effect of S-R correspondence was not significant $[F(1,15)<1]$. That is, there was no overall Simon effect. Most impor-

Table 1

Reaction Times (RTs, in Milliseconds) and Percentages of Errors (PEs), As a Function of Stimulus-Response (S-R) Mapping and Spatial S-R Correspondence, Observed in Experiment $1(N=16) \mathrm{m}$

\begin{tabular}{|c|c|c|c|c|c|c|}
\hline & \multicolumn{2}{|c|}{$\begin{array}{c}\text { Compatible } \\
\text { S-R } \\
\text { Mapping }\end{array}$} & \multicolumn{2}{|c|}{$\begin{array}{c}\text { Incompatible } \\
\text { S-R } \\
\text { Mapping }\end{array}$} & \multicolumn{2}{|c|}{$\begin{array}{c}\text { Mapping } \\
\text { Effect }\end{array}$} \\
\hline & RT & $\mathrm{PE}$ & RT & PE & RT & PE \\
\hline Location corresponding (co) & 539 & 2.8 & 626 & 11.4 & 87 & 8.6 \\
\hline Location noncorresponding (nc) & 561 & 4.2 & 608 & 6.6 & 47 & 2.4 \\
\hline Simon effect $(\mathrm{nc}-\mathrm{co})$ & 22 & 1.4 & -18 & -4.8 & & \\
\hline
\end{tabular}


tant, however, there was a significant mapping $\times \mathrm{S}-\mathrm{R}$ correspondence interaction $\left[F(1,15)=107.07, M S_{\mathrm{e}}=\right.$ 59.26, $p<.001]$. With the compatible mapping, RTs were shorter in spatially corresponding than in spatially noncorresponding conditions (539vs. $561 \mathrm{msec})[t(15)=$ $4.47, p<.001]$. By contrast, with the incompatible mapping, RTs were longer in spatially corresponding than in spatially noncorresponding conditions (626 vs. $608 \mathrm{msec}$ ) $[t(15)=3.39, p<.01]$. Fifteen participants showed the normal Simon effect with compatible mapping; 13 participants showed the reversed Simon effect with the incompatible mapping.

Percentage of errors. The percentages of response errors observed in the different experimental conditions were also subjected to an ANOVA for repeated measurements designs with mapping and $\mathrm{S}-\mathrm{R}$ correspondence as within-subjects variables. The results perfectly mirrored those of the RT analysis. The main effect of mapping was significant $\left[F(1,15)=41.56, M S_{\mathrm{e}}=11.68, p<.001\right]$. The participants responded more accurately with the compatible than with the incompatible mapping (3.5\% vs. $9.0 \%)$. The main effect of S-R correspondence was not significant $[F(1,15)=2.02, p=.18]$. That is, overall Simon effects occurred neither in RTs nor in errors. There was, however, again a significant mapping $\times \mathrm{S}-\mathrm{R}$ correspondence interaction $\left[F(1,15)=7.27, M S_{\mathrm{e}}=20.96, p<.05\right]$. With the compatible mapping, the participants responded somewhat more accurate in spatially corresponding than in spatially noncorresponding conditions ( $2.8 \%$ vs. $4.2 \%)$ $[t(15)=1.33, p=.20]$. By contrast, with the incompatible mapping, the participants responded less accurately in spatially corresponding than in spatially noncorresponding conditions $(11.4 \%$ vs. $6.6 \%)[t(15)=2.30, p<.05]$.

\section{Discussion}

The results of Experiment 1 replicated the pattern of results first reported by Hedge and Marsh (1975). When the participants responded to a finger name by moving the denoted finger (compatible S-R mapping), a regular Simon effect occurred: Responses were faster and the error rate lower with spatial correspondence than with spatial noncorrespondence between the irrelevant stimulus location and the irrelevant response location. By contrast, when the participants responded to a finger name by moving the opposite finger (incompatible S-R mapping), an inverted Simon effect occurred: Responses were slower and the error rate higher with spatial correspondence than with spatial noncorrespondence between the irrelevant stimulus location and the irrelevant response location.

The results of Experiment 1 are incompatible with an account in terms of S-S congruity (e.g., Simon \& Sudalaimuthu, 1979), because there is no consistent relationship between finger names and spatial locations. In addition, the results of Experiment 1 cannot be explained on the basis of the original display-control arrangement correspondence hypothesis (Simon et al., 1981), because there is no visual similarity between nonspatial stimulus and response features. However, the results of Experiment 1 are consistent with the logical-recoding hypothesis. According to this account, the participants used the identity rule (i.e., use the finger denoted by the word) for selecting the correct response with the compatible S-R mapping, whereas the participants used the reversal rule (i.e., use the finger opposite to that denoted by the word) for response selection with the incompatible S-R mapping. Moreover, in accordance with Hedge and Marsh (1975) and De Jong et al. (1994), we assume that the rule used for response selection was inadvertently applied to the irrelevant S-R dimension, producing a regular Simon effect with the compatible mapping and a reversed Simon effect with the incompatible mapping.

Although the results of Experiment 1 are inconsistent with the original version of the display-control arrangement correspondence hypothesis that focuses on the spatial arrangement of physical (i.e., visible) stimulus and response features, an extension of the hypothesis to the conceptual level might explain the findings. ${ }^{1}$ According to the extended hypothesis, responses are facilitated if conceptually matching stimulus and response features appear at the same relative location, as opposed to when they appear at opposite locations. The results of Experiment 1 do not allow one to decide between the logical-recoding hypothesis and the display-control arrangement correspondence hypothesis on a conceptual level. Thus, it is important to determine whether display-control arrangement correspondence exerts its effects in isolation.

\section{EXPERIMENT 2}

The purpose of Experiment 2 was to study the effect of display-control arrangement correspondence with regard to color on the effect of spatial S-R correspondence without any effect of logical-recoding operations. In Experiment 2, participants responded to the shape of a geometrical figure (i.e., diamond vs. square; see Figure 3) by pressing a left or a right key. We independently varied two S-R correspondence relationships. First, we varied spatial S-R correspondence by presenting the stimulus randomly to the left or right of fixation. Second, we varied color S-R correspondence by presenting the stimulus randomly in green or in red color, and the participants wore a green glove on one hand and a red glove on the other hand throughout the experiment. In order to force the participants to attend to stimulus color, as in the original experiments by Hedge and Marsh (1975) and Simon et al. (1981), we had the stimulus appear in a third color (blue) on $20 \%$ of the trials in each experimental block. These trials were no-go trials, on which the participants were instructed to refrain from responding.

The independent variation of spatial S-R correspondence and of color S-R correspondence allows for different patterns of results. If display-control arrangement correspondence was effective, we should expect spatial $\mathrm{S}-\mathrm{R}$ correspondence and color $\mathrm{S}-\mathrm{R}$ correspondence to have interactive effects. That is, performance should be better when both relationships are corresponding (e.g., green-left $\mathrm{S} \rightarrow$ green-left R [Figure 3A]) or when both relationships are noncorresponding (e.g., red-right $\mathrm{S} \rightarrow$ green-left R [Figure 3G]) than when one relationship is corresponding and the other one noncorresponding 


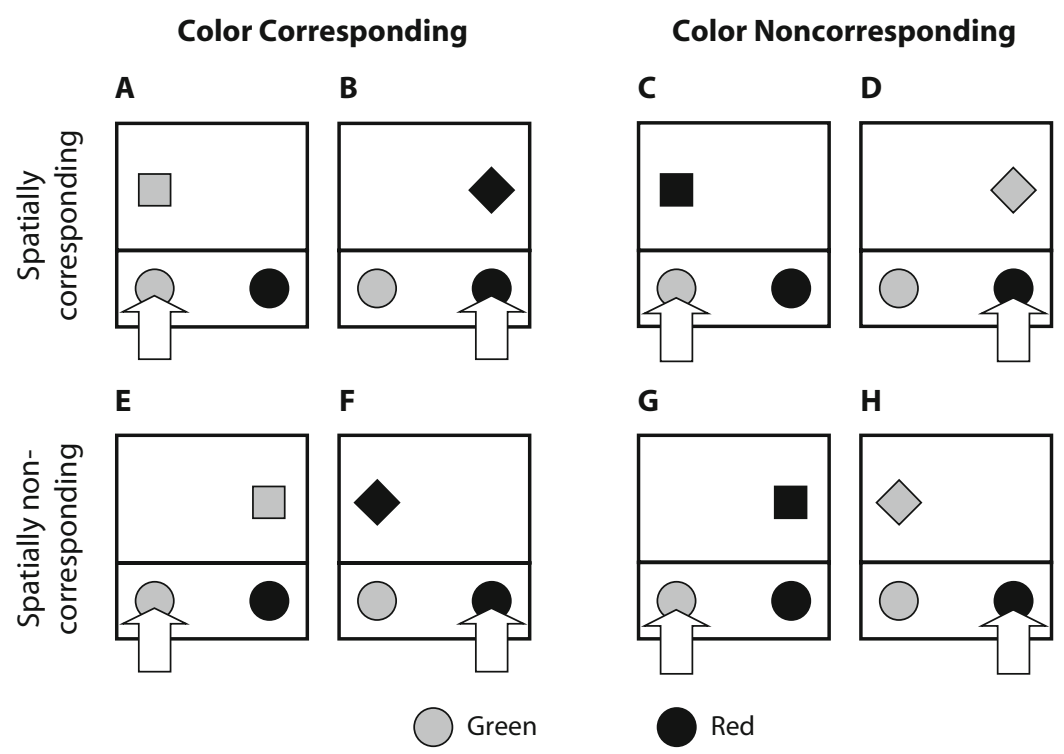

Figure 3. Different experimental conditions in the present Experiment 2. Participants responded to stimulus shape (diamond vs. square) with left/right keypresses. In this example, the participants responded with the left-green hand to the square and with the right-red hand to the diamond. Hand color resulted from wearing colored gloves. The stimuli appeared unpredictably in green or red color at different locations (left vs. right).

(e.g., red-left $\mathrm{S} \rightarrow$ green-left R [Figure 3C], or green-right $\mathrm{S} \rightarrow$ green-left $\mathrm{R}$ [Figure 3E]). By contrast, if displaycontrol arrangement correspondence was not effective in the present task, we should observe only main effects of spatial S-R correspondence and of color S-R correspondence but no interaction.

Please note that neither logical recoding nor S-S congruity could play a role in Experiment 2. With regard to logical recoding, the mapping of stimulus shape (diamond vs. square) to the location and/or color of responses does not seem to afford logical recoding of the S-R rules. With regard to S-S congruity, the two shapes do not appear to have strong associations with particular locations or colors.

\section{Method}

Participants. Twenty-four new volunteers (18 of them female, 6 male), mostly students at the Friedrich Alexander University with a mean age of 23.5 years (range, 20-30 years), received money (€3) or course credit for participation.

Apparatus and Stimuli. The apparatus from Experiment 1 was also used for Experiment 2. A plus sign served as fixation at screen center. A diamond and a square subtending approximately $20 \times$ $20 \mathrm{~mm}$ (about $1.9^{\circ} \times 1.9^{\circ}$ ) served as stimuli. A stimulus was presented $70 \mathrm{~mm}$ (approximately $6.7^{\circ}$ ) to either the left or the right of fixation. The diamond or square was presented in blue, green, or red color (i.e., the area covered by the stimulus was colored) on a black background. The participants' task was to respond to the shape of a green or red stimulus by pressing either the left or the right control key on a standard computer keyboard and to refrain from responding to a blue stimulus. The participants operated the two keys with the index fingers of the two hands. Finally, during the experiment, 12 participants wore a green glove on the left hand and a red glove on the right hand; 12 participants wore a red glove on the left hand and a green glove on the right hand. Note that, with a similar setup, we had successfully replicated the results observed by Hedge and Marsh (1975) in a previous study (Wühr, 2004).

Procedure. At the beginning of the experiment, instructions appeared on the screen, and the participants could read them at their leisure. The participants were informed that a diamond or square would unpredictably appear to the left or right of fixation in one of three different colors (blue, green, or red). Moreover, the participants were told to respond to the shape of a green or red stimulus and to refrain from responding to a blue stimulus. Half of the participants responded to the (green or red) diamond by pressing the left key and to the (green or red) square by pressing the right key. The other half of the participants responded on the basis of the opposite S-R mapping.

Having read the instructions, each participant practiced for 2 blocks of 22 trials each. In the experimental phase, the participants worked through 10 blocks of 22 trials each. The first 2 trials in each block were warm-up trials and were not recorded. Each block contained 16 go stimuli in green or red color ( 2 repetitions $\times$ 2 stimulus shapes $\times 2$ stimulus colors $\times 2$ stimulus locations) and 4 no-go stimuli in blue color ( 2 stimulus shapes $\times 2$ stimulus locations). Thus, there were $80 \%$ go and $20 \%$ no-go trials. The sequence of events in an experimental trial was the same as in Experiment 1. Participants could take short rests between blocks. The whole experiment took about $15 \mathrm{~min}$.

Design. Experiment 2 rested on a $2 \times 2$ within-subjects design. The first factor concerned spatial $S-R$ correspondence between the location of the stimulus on the screen and the relative location of the response key (or hand). The second factor concerned color $S-R$ correspondence between the color of the stimulus and the color of the glove worn by the responding hand. Dependent variables were RTs of correct responses and the percentages of errors (PEs). Note that each experimental block also contained four no-go trials with blue stimuli that forced the participants to attend to stimulus color.

\section{Results}

To eliminate outliers, we removed all trials on which RTs differed from the individual mean by more than three 
Table 2

\begin{tabular}{|c|c|c|c|c|c|c|}
\hline & \multicolumn{2}{|c|}{$\begin{array}{c}\text { Color } \\
\text { Corresponding }\end{array}$} & \multicolumn{2}{|c|}{$\begin{array}{c}\text { Color } \\
\text { Noncorresponding }\end{array}$} & \multicolumn{2}{|c|}{$\begin{array}{c}\text { Color } \\
\text { Correspondence } \\
\text { Effect }\end{array}$} \\
\hline & RT & PE & RT & PE & RT & $\mathrm{PE}$ \\
\hline Location corresponding (co) & 520 & 3.0 & 532 & 4.3 & 12 & 1.3 \\
\hline Location noncorresponding (nc) & 533 & 4.3 & 541 & 5.0 & 9 & 0.7 \\
\hline Simon effect $(n c-c o)$ & 13 & 1.3 & 9 & 0.7 & & \\
\hline
\end{tabular}

Note-The differences between noncorresponding and corresponding conditions with regard to color (rightmost column) and with regard to location (last line) are also given.

standard deviations. This procedure eliminated $1.0 \%$ of the trials. The mean error rate in the 40 no-go trials was $8.8 \%(S D=9.2)$, ranging from $0 \%$ to $35 \%$. Excluding the participant with the highest response rate in the no-go trials does not alter the pattern of results. RTs and percentages of errors observed in Experiment 2 are listed in Table 2. Initial analyses showed that the mapping of stimulus shapes to response hands had no effects on performance (both main effects had $F$ values $<1$; all interactions had $F$ values $<1.6$ and $p$ values $>.20$ ).

Reaction times. RTs from error-free trials were subjected to an ANOVA with spatial correspondence and color correspondence as within-subjects variables. The main effect of spatial correspondence was significant $\left[F(1,23)=8.38, M S_{\mathrm{e}}=339.15, p<.01\right]$. The participants responded more quickly with corresponding rather than with noncorresponding colors of the stimulus and the responding hand $(527$ vs. $537 \mathrm{msec})$. That is, a spatial Simon effect of $11 \mathrm{msec}$ was obtained. The main effect of color correspondence was also significant $[F(1,23)=$ $\left.9.37, M S_{\mathrm{e}}=258.10, p<.01\right]$. The participants responded more quickly with correspondence than with noncorrespondence of the stimulus and the responding hand $(527$ vs. $537 \mathrm{msec})$. That is, a color correspondence effect of $10 \mathrm{msec}$ was obtained. Most important, however, the two-way interaction between color correspondence and spatial correspondence was far from significant $(F<1)$.

Percentages of errors. The percentages of response errors observed in the different experimental conditions were also subjected to an ANOVA for repeated measurements designs, with spatial correspondence and color correspondence as within-subjects variables. Neither the main effect of location correspondence $[F(1,23)=1.78$, $\left.M S_{\mathrm{e}}=13.18, p=.20\right]$ nor the main effect of color correspondence $\left[F(1,23)=1.12, M S_{\mathrm{e}}=21.06, p=.30\right]$ was significant. The two-way interaction also failed to reach significance $(F<1)$.

\section{Discussion}

The results of Experiment 2 revealed negative evidence for the notion of display-control arrangement correspondence when effects of logical-recoding operations and S-S congruity were prevented. The independent variation of spatial S-R correspondence and of color S-R correspondence revealed independent main effects of location correspondence (i.e., a Simon effect) and of color correspondence in RTs, indicating that both relationships were processed. Most important, however, there was no interaction between these two variables, and this finding stands in contrast to the prediction of the display-control arrangement correspondence hypothesis.

\section{GENERAL DISCUSSION}

The present study investigated the effects of logicalrecoding operations and display-control arrangement correspondence on response selection. These variables have been proposed as alternative explanations for the so-called Hedge and Marsh reversal of the Simon effect. Previous studies, however, did not provide clear evidence for either of these variables (see Proctor \& Vu, 2006, for a review). Discovering the source(s) of the reversal of the Simon effect is important both for theoretical and for practical reasons, because it represents a notable exception to the general rule that performance is superior with spatially corresponding than with spatially noncorresponding S-R arrangements.

Experiment 1 provided evidence for an effect of logicalrecoding operations on response selection. The participants responded compatibly or incompatibly to finger names that appeared randomly at left or right locations. The compatible mapping revealed a normal Simon effect of spatial correspondence between word and finger location; the incompatible mapping produced a reversed Simon effect. These findings support the application of logical-recoding rules to the relevant and the irrelevant $\mathrm{S}-\mathrm{R}$ dimension, because S-S congruity (e.g., Simon \& Sudalaimuthu, 1979) and display-control arrangement correspondence on a visual level (e.g., Simon et al., 1981) did not play a role in Experiment 1. S-S congruity was not effective, because a finger is not associated with a particular relative location, due to the symmetric arrangement of fingers on the left and right hands. Similarly, display-control arrangement correspondence was ineffective because finger words and fingers do not share visible features. It should be noted, however, that the results of Experiment 1 are still compatible with the notion of display-control arrangement correspondence on a conceptual level. According to this version of the hypothesis, responses are facilitated if conceptually matching stimulus and response features appear at the same relative location, as opposed to when they appear at opposite locations. 
Experiment 2 failed to provide support for an effect of display-control arrangement correspondence on response selection when the application of logical-recoding rules was prevented. In this experiment, the participants selected a response with a colored hand on the basis of stimulus shape, and we independently varied S-R correspondence with regard to location and color. We observed significant main effects of spatial S-R correspondence (i.e., a Simon effect) and of color-based S-R correspondence, but these two variables did not interact. In particular, there was no reversal of the spatial Simon effect with noncorrespondence between the color of the stimulus and the color of the responding hand, disconfirming the predictions of the display-control arrangement correspondence hypothesis. Thus, the results of Experiment 2 suggest that displaycontrol arrangement correspondence (with regard to color) is not able to modulate the effect of spatial S-R correspondence.

The finding from Experiment 2 that multiple codes can produce independent (i.e., additive) S-R correspondence effects is consistent with previous reports in the literature (e.g., Lamberts, Tavernier, \& d'Ydewalle, 1992; Llereas, Moore, \& Mordkoff, 2004; Wühr, Biebl, \& Ansorge, 2008). For example, several studies have shown that S-R correspondence with regard to different spatial frames of reference can produce independent Simon effects (e.g., Ansorge \& Wühr, 2004; Lamberts et al., 1992; Llereas et al., 2004). More important for the present study, however, are more recent reports demonstrating that spatial and nonspatial feature dimension can also produce independent S-R correspondence effects. For example, Wühr et al. (2008) observed independent Simon effects for spatial location and for numerosity when stimulus tones and the required keypress responses varied independently in number and location.

How can the independent effects of multiple codes on performance be explained? According to the response discrimination account (see Ansorge \& Wühr, 2004; Wühr et al., 2008), Simon effects arise from the interaction between stimulus location codes and response location codes in working memory if response location is used for representing and discriminating between alternative responses in working memory. This principle can easily be extended to multiple dimensions in assuming that multiple stimulus features can produce Simon effects if the task affords (or requires) the inclusion of multiple features in the working memory representation of the alternative responses. In particular, the task used in Experiment 2 afforded the inclusion of both color and location into the working memory representation of the alternative responses, because there was a consistent relationship between response color and response location. Thus, given that the participants processed (i.e., attended to) stimulus color and stimulus location, stimulus location and stimulus color codes were formed in working memory and independently activated those S-R rules that contained a matching (or corresponding) feature code.

Together, the results of our experiments support the notion that logical recoding of S-R rules, and not displaycontrol arrangement correspondence, is able to modulate the effects of spatial S-R correspondence and to produce the Hedge and Marsh reversal of the Simon effect. This result has important theoretical implications for our understanding of response selection. First, the results of Experiment 1 provide the most direct evidence for the fact that participants instantaneously derive generalized response selection rules (i.e., the identity or the reversal rule) from specific $\mathrm{S}-\mathrm{R}$ rules (e.g., green $\mathrm{S} \rightarrow$ green $\mathrm{R}$, red $\mathrm{S} \rightarrow$ red R). Second, the results also demonstrate that the generalized response selection rules are applied not only to the relevant S-R dimension (e.g., color, shape), but also to an irrelevant $\mathrm{S}-\mathrm{R}$ dimension (e.g., location). The second result extends previous findings demonstrating that specific S-R rules (e.g., letter $\mathrm{A} \rightarrow$ left response, letter $\mathrm{B} \rightarrow$ right response) are also applied to irrelevant stimuli that are presented in the vicinity of the relevant stimulus (e.g., Eriksen \& Eriksen, 1974).

To conclude, the present study adds to the growing number of studies suggesting that the classical view of spatial S-R correspondence as a highly automatic, stimulus-driven phenomenon is inadequate (e.g., Ansorge \& Wühr, 2004; Hommel, 1993; Valle-Inclan \& Redondo, 1998; Wühr et al., 2008). Rather, top-down processes such as the derivation of abstract response selection rules from specific S-R rules appear to play a major role in how irrelevant stimulus information activates corresponding response representations.

\section{AUTHOR NOTE}

We thank Alexandra Kopp and Inga Reismann for running the participants. Moreover, we thank Bernhard Hommel and Robert Proctor for commenting on a previous version of the manuscript. Correspondence concerning this article should be addressed to P. Wühr, TU Dortmund, Institut für Psychologie, Emil-Figge-Strasse 50, 44227 Dortmund, Germany (e-mail: wuehr@fk14.tu-dortmund.de).

$$
\begin{array}{r}
\text { Note-Accepted by the previous editorial team, } \\
\text { when Thomas H. Carr was Editor. }
\end{array}
$$

\section{REFERENCES}

AnsORgE, U., \& WÜHR, P. (2004). A response-discrimination account of the Simon effect. Journal of Experimental Psychology: Human Perception \& Performance, 30, 365-377. doi:10.1037/0096-1523.30.2.365

AREND, U., \& WANDMACHER, J. (1987). On the generality of logical recoding in spatial interference tasks. Acta Psychologica, 65, 193-210. doi:10.1016/0001-6918(87)90049-7

De Jong, R., Liang, C.-C., \& Lauber, E. (1994). Conditional and unconditional automaticity: A dual-process model of effects of spatial stimulus-response correspondence. Journal of Experimental Psychology: Human Perception \& Performance, 20, 731-750. doi:10.1037/0096-1523.20.4.731

Eriksen, B. A., \& ERIKSEN, C. W. (1974). Effects of noise letters upon the identification of a target letter in a nonsearch task. Perception \& Psychophysics, 16, 143-149.

HasbroucQ, T., \& GuiARD, Y. (1991). Stimulus-response compatibility and the Simon effect: Toward a conceptual clarification. Journal of Experimental Psychology: Human Perception \& Performance, 17, 246-266. doi:10.1037/0096-1523.17.1.246

Hedge, A., \& Marsh, N. W. A. (1975). The effect of irrelevant spatial correspondence on two-choice response-time. Acta Psychologica, 39, 427-439. doi:10.1016/0001-6918(75)90041-4

Hommel, B. (1993). Inverting the Simon effect by intention: Determinants of direction and extent of effects of irrelevant spatial information. Psychological Research, 55, 270-279. doi:270-279. 10.1007/ BF00419687 
Hommel, B., \& Prinz, W. (1997). Theoretical issues in stimulusresponse compatibility. Amsterdam: Elsevier.

Kornblum, S., HasbroucQ, T., \& Osman, A. (1990). Dimensional overlap: Cognitive basis for stimulus-response compatibility-a model and taxonomy. Psychological Review, 97, 253-270. doi:253270. 10.1037/0033-295X.97.2.253

Kramer, A. F., \& Jacobson, A. (1991). Perceptual organization and focused attention: The role of objects and proximity in visual processing. Perception \& Psychophysics, 50, 267-284.

Lamberts, K., Tavernier, G., \& D'Ydewalle, G. (1992). Effects of multiple reference points in spatial stimulus-response compatibility. Acta Psychologica, 79, 115-130. doi:10.1016/0001-6918(92)90028-C

Llereas, A., Moore, C. M., \& MordKoff, J. T. (2004). Looking for the source of the Simon effect: Evidence for multiple codes. American Journal of Psychology, 117, 531-542. doi:10.2307/4148990

Lu, C.-H., \& Proctor, R. W. (1994). Processing of an irrelevant location dimension as a function of the relevant stimulus dimension. Journal of Experimental Psychology: Human Perception \& Performance, 2, 286-298. doi:10.1037/0096-1523.20.2.286

Proctor, R. W., \& Pick, D. F. (2003). Display-control arrangement correspondence and logical recoding in the Hedge and Marsh reversal of the Simon effect. Acta Psychologica, 112, 259-278. doi:10.1016/ S0001-6918(02)00125-7

Proctor, R. W., \& VU, K.-P. L. (2006). Stimulus-response compatibility principles: Data, theory, and practice. Boca Raton, FL: Taylor \& Francis.

Simon, J. R., \& RudeLL, A. P. (1967). Auditory S-R compatibility: The effect of an irrelevant cue on information processing. Journal of Applied Psychology, 51, 300-304. doi:10.1037/h0020586

Simon, J. R., Sly, P. E., \& VilapaKKam, S. (1981). Effect of compatibility of S-R mapping on reactions toward the stimulus source. Acta Psychologica, 47, 63-81. doi:10.1016/0001-6918(81)90039-1

Simon, J. R., \& Sudalaimuthu, P. (1979). Effects of S-R mapping and response modality on performance in a Stroop task. Journal of Experimental Psychology: Human Perception \& Performance, 5, 176-187. doi:10.1037/0096-1523.5.1.176

VALLE-INCLAN, F., \& REDONDO, M. (1998). On the automaticity of ipsilateral response activation in the Simon effect. Psychophysiology, 35, 366-371. doi:10.1017/S0048577298960917

WÜHR, P. (2004). Sequential modulations of logical-recoding operations in the Simon task. Experimental Psychology, 51, 98-108. doi:10.1027/1618-3169.51.2.98

WÜHr, P., BIEBL, R., \& ANSORgE, U. (2008). The impact of stimulus and response variability on S-R correspondence effects. Journal of Experimental Psychology: Learning, Memory, \& Cognition, 34, 533545. doi:10.1037/0278-7393.34.3.533

Wühr, P., Biebl, R., Umiltà, C., \& Müsseler, J. (in press). Perceptual and attentional factors in encoding irrelevant spatial information. Psychological Research. doi:10.1007/s00426-008-0160-8

WÜHR, P., \& WASZAK, F. (2003). Object-based attentional selection can modulate the Stroop effect. Memory \& Cognition, 31, 983-994.

Zhang, H., Zhang, J., \& Kornblum, S. (1999). A parallel distributed processing model of stimulus-stimulus and stimulus-response compatibility. Cognitive Psychology, 38, 386-432. doi:10.1006/ cogp.1998.0703

ZorZi, M., \& Umiltà, C. (1995). A computational model of the Simon effect. Psychological Research, 58, 193-205. doi:10.1007/ BF00419634

\section{NOTE}

1. We thank Robert Proctor for suggesting this possibility.

(Manuscript received November 13, 2007; revision accepted for publication September 15, 2008.) 